\title{
ESTUDO DO PROCESSO DE PURIFICAÇÃO DO XILITOL OBTIDO POR ROTA FERMENTATIVA UTILIZANDO A LEVEDURA Kluyveromyces marxianus CCA510
}

\author{
T. L. de ALBUQUERQUE ${ }^{1}$, L. F. SERAFIM ${ }^{1}$, M. R. A. ARCANJO ${ }^{1}$, J. E. M. JUNIOR ${ }^{1}$, I. J. \\ SILVA Jr ${ }^{1}$ e M. V. P. ROCHA ${ }^{1}$ \\ ${ }^{1}$ Universidade Federal do Ceará, Departamento de Engenharia Química \\ E-mail para contato: tiagotla1@gmail.com; valderez.rocha@ufc.br
}

\begin{abstract}
RESUMO - A via biotecnológica é uma alternativa para a produção de xilitol, poliol com utilização na indústria alimentícia e farmacêutica. Contudo, são escassos os estudos que visem à recuperação dessa substância a partir dos meios de cultivo microbiano. Assim, objetivou-se avaliar o processo de purificação do xilitol obtido por processo biotecnológico utilizando a levedura Kluyveromyces marxianus CCA510 e meio semissintético. Após o bioprocesso, o meio de cultivo livre de células foi tratado avaliando-se diferentes adsorventes (resinas de troca iônica) e posteriormente concentrado para utilização no estudo da cristalização. Dois antissolventes (etanol e isopropanol) foram analisados na cristalização e os resultados não apresentaram diferença significativa no rendimento dos cristais. As melhores condições de tratamento e cristalização produziram cristais de xilitol com pureza de $94 \%$, rendimento de cristalização de $60 \%$ e através de análises de microscopia, os cristais obtidos apresentaram semelhantes ao xilitol padrão.
\end{abstract}

\section{INTRODUÇÃO}

$\mathrm{O}$ xilitol $\left(\mathrm{C}_{5} \mathrm{H}_{12} \mathrm{O}_{5}\right)$ é um poliol que apresenta diversas aplicações alimentícias, farmacêuticas e odontológicas com ação anticariogênica (Soleimani e Tabil, 2014) e com poder adoçante semelhante ao da sacarose e superior ao de polióis comuns, além de ter valor calórico reduzido de 2,4 kcal/g (Burneo, 2013). Em larga escala, este poliol é produzido por hidrogenação da D-xilose, na presença de ligas de $\mathrm{Ni}$ e $\mathrm{Al}$, sob o uso de altas temperaturas e pressões, aumentando o custo do produto final (Ahmad et al., 2013). Diante das desvantagens relacionadas com o processo químico de obtenção do xilitol, surgiu a necessidade de se desenvolver tecnologias alternativas e mais atrativas para sua obtenção, como a via microbiológica (Sarrouh, 2009).

O uso de xilitol em produtos industrializados já foi aprovado em mais de quarenta países, principalmente em indústrias alimentícias, farmacêuticas e de cosméticos. No Brasil, as indústrias estão começando a incluir o xilitol na formulação de produtos, atraídas pelo seu efeito refrescante e, sobretudo, pela sua ação anticariogênica.

Embora a maior parte dos estudos de produção de xilitol sejam focados no tratamento de hidrolisado e na bioconversão da xilose, estudos recentes sobre o processo de cristalização do xilitol são citados na literatura (Martínez et al., 2009; Misra et al., 2011; Wei et al., 2010). A cristalização é um processo de separação, que consiste no surgimento e no crescimento de partículas sólidas no meio, provocados por uma instabilidade na solução (Derenzo, 1994). 
O processo de separação de um produto representa ao redor de 20-80 \% do custo do produto, sendo que cerca de $70 \%$ dos produtos comercializados são sólidos (Martínez, 2005), tornando-se assim importante o aumento do conhecimento da ciência da cristalização, devido ao aumento das exigências de perfeição estrutural, homogeneidade e controle de defeitos dos cristais (Misra et al., 2011). Este processo facilita os passos seguintes da produção tais como filtração, lavagem e secagem, melhorando a aparência do produto, aspecto importante para a aceitação do consumidor (Belter et al., 1988).

A cristalização é uma etapa chave para obtenção de xilitol, uma vez que, em muitos aspectos, ela determina o rendimento e a qualidade do produto. Para escolher solventes adequados e para a concepção de um cristalizador optimizado, é necessário conhecer o efeito de solventes na solubilidade e cinética da cristalização do xilitol (Hao et al., 2006; Misra et al., 2011). Dessa maneira, estudos que busquem a avaliação das propriedades de cristalização desse composto são necessários para conhecimento e otimização do processo de produção. Nesse contexto, o presente trabalho visa avaliar o processo de cristalização de xilitol produzido a partir de meio semissintético por Kluyveromyces marxianus CCA510.

\section{MATERIAL E MÉTODOS}

\subsection{Curvas de Solubilidade do Xilitol PA}

Curvas de solubilidade foram construídas a partir de soluções de concentrações conhecidas, de xilitol em água, água-etanol (1:1) e água-isopropanol (1:1), sendo esta proporção selecionada de acordo com os estudos realizados por Wei et al. (2010). As soluções foram aquecidas até $60{ }^{\circ} \mathrm{C}$ para garantir a completa dissolução dos cristais e então resfriadas, sobre constante agitação, até a formação do primeiro cristal visível a olho nu. Tais pontos de saturação foram anotados, gerando um gráfico de concentração versos temperatura. Esse procedimento foi conduzido em triplicata.

\subsection{Produção do Xilitol por Processo Biotecnológico}

Micro-organismo e condição de cultivo: O micro-organismo e composição do meio foram baseados no estudo realizado por Albuquerque (2014). O micro-organismo utilizado para a produção do xilitol foi Kluyveromyces marxianus CCA 510 pertencente ao Laboratório de Microbiologia Agrícola da UFSCar. Os ensaios foram realizados em batelada em biorreator (TECNAL, modelo TEC-BIO 1.5) com dorna de 1,0 L contendo $750 \mathrm{~mL}$ de meio (xilose, 40 $\mathrm{g} / \mathrm{L}$; extrato de levedura, $10 \mathrm{~g} / \mathrm{L}$ e $\left.\left(\mathrm{NH}_{4}\right)_{2} \mathrm{SO}_{4}, 5 \mathrm{~g} / \mathrm{L}\right)$. $\mathrm{O}$ bioprocesso de produção de xilitol foi conduzido a $30{ }^{\circ} \mathrm{C}$, aeração de $1,3 \mathrm{vvm}$ e $150 \mathrm{rpm}$ por $72 \mathrm{~h}$. Após o cultivo, as células foram separadas do meio fermentado por centrifugação a $10.000 \mathrm{~g}$ por 15 minutos a $4{ }^{\circ} \mathrm{C}$. O meio cultivado livre de células foi utilizado nas etapas posteriores para a separação do xilitol. Foram medidas as concentrações de xilitol produzido e xilose presente no sobrenadante.

\subsection{Processo de Recuperação do Xilitol}

Tratamento do meio bioprocessado utilizando resinas de troca iônica: O meio cultivado livre de células foi submetido a um tratamento para separação de substâncias interferentes a etapa de cristalização, por cromatografia, em colunas cromatográficas da Superfomance (Gotec 
Labortechnic). Para os experimentos foram utilizadas a resina Dowex® Monosphere $99 \mathrm{Ca}$ (Sigma-Aldrich) com diâmetro de partícula de $320 \mu \mathrm{m}$, nas dimensões $2,5 \mathrm{~cm} \times 11,5 \mathrm{~cm}$ e a resina Diaion® UBK 555(Sigma-Aldrich) com diâmetro de partícula de 220 ${ }^{\circledR}$ m, nas dimensões $2,5 \mathrm{~cm} \times 10 \mathrm{~cm}$. Primeiramente o meio foi tratado com a resina Dowex monosphere 99Ca (Sigma-Aldrich e, em seguida com a resina Diaion UBK555 (Sigma-Aldrich), bombeadas por uma bomba de deslocamento positivo com vazão de $4 \mathrm{~mL} \cdot \mathrm{min}^{-1}$.

Concentração do meio bioprocessado e tratado: Após o tratamento inicial, o meio foi concentrado em evaporador rotativo (Quimis, Modelo Q344B) a $65{ }^{\circ} \mathrm{C}$ até obter uma concentração desejada.

Cristalização: O processo de cristalização ocorreu em um arranjo paralelo de dois reatores encamisados de $100 \mathrm{~mL}$, ligados a um banho termostatizado, sobre agitação de $150 \mathrm{rpm}$. Nessa etapa foi avaliada a influência de dois álcoois, etílico e isopropílico, no processo de cristalização do xilitol. O meio cultivado, tratado e concentrado foi dividido igualmente entre os dois cristalizadores e os álcoois foram adicionados na proporção de 1:1 separadamente em cada cristalizador. $\mathrm{O}$ processo iniciou-se a $50{ }^{\circ} \mathrm{C}$, permanecendo por 10 minutos e em seguida o sistema foi resfriado até uma temperatura de $5{ }^{\circ} \mathrm{C}$ por um tempo total de $24 \mathrm{~h}$. Quatro cristais de xilitol (com 99,9\% de pureza, marca Sigma) foram adicionados nos respectivos pontos de saturação, $35{ }^{\circ} \mathrm{C}$ no cristalizador com álcool etílico e $43{ }^{\circ} \mathrm{C}$ no cristalizador com álcool isopropílico, para facilitar o processo de nucleação. Após o término do processo de cristalização, filtrou-se para separar os cristais do sobrenadante, sendo analisado o sobrenadante e os cristais foram submetidos a análise de pureza e microscopia. Para examinar a eficiência do sistema de cristalização, foi realizado o mesmo procedimento utilizando-se xilitol com pureza de 99,9\% (Sigma), a partir de uma solução com fração mássica de 44,7\% m/v de xilitol (álcool etílico e água (1:1) no primeiro cristalizador e álcool isopropílico e água (1:1) no segundo cristalizador).

\subsection{Determinação da Pureza do Xilitol}

A aparência dos cristais de xilitol foi avaliada por microscopia ótica com ampliação de 440x. A pureza dos cristais foi determinada por Cromatografia Líquida de Alta Eficiência (Waters, Milford, MA, E.U.A.), pesando-se uma determinada massa dos cristais dissolvendo em $5 \mathrm{~mL}$ de água deionizada e analisando em CLAE conforme citado no item 2.5.

\subsection{Métodos Analíticos}

Xilose, glicose, xilitol, ácido acético e etanol foram analisados por Cromatografia Líquida de Alta Eficiência (CLAE) utilizando um sistema de CLAE (Waters, Milford, MA, E.U.A.) equipado com um detector de índice de refração Waters e com uma coluna Aminex HPX-87H (Bio Rad, Hercules, CA, USA). O eluente foi $\mathrm{H}_{2} \mathrm{SO}_{4} 5 \mathrm{mmol} / \mathrm{L}$ em água deionizada (MilliQ Simplicity 185 , Millipore, Billerica, MA) com vazão de $0,5 \mathrm{~mL} / \mathrm{min}$ a $65^{\circ} \mathrm{C}$. O volume de cada amostra foi de $20 \mu \mathrm{L}$.

\section{RESULTADOS E DISCUSSÃO}

\subsection{Curva de Solubilidade}

A Figura 1 mostra as curvas de solubilidade do xilitol em três solventes diferentes: água, 
água:etanol (1:1) e água:isopropanol (1:1). Tais curvas foram construídas para o conhecimento dos graus de supersaturação que o sistema pode atingir e para auxiliar nos devidos balanços de massa que serão realizados durante o processo de cristalização.

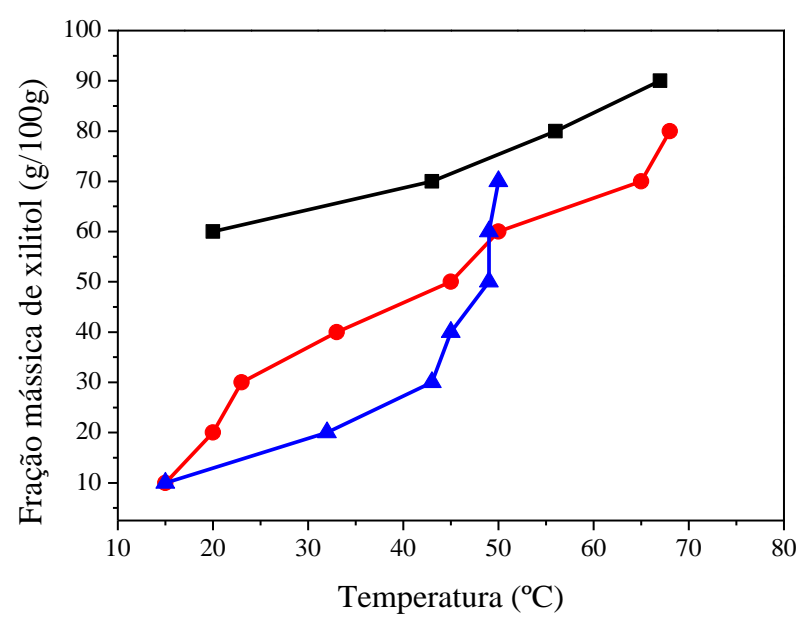

Figura 1: Curva de solubilidade representando a fração mássica de xilitol dissolvida em diferentes sistemas. ( $\bullet$ água, $(\boldsymbol{\Delta})$ água e etanol e (•) água e isopropanol.

Observando a Figura 1, nota-se que o xilitol é solúvel nos três solventes a altas temperaturas, porém, em temperaturas, próximas de $20{ }^{\circ} \mathrm{C}$, observa-se uma baixa solubilidade nos álcoois etanol e isopropanol, revelando uma ação anti-solvente.

A escolha do método de cristalização quando se parte de uma solução, depende da inclinação da curva de solubilidade versus temperatura. A supersaturação pode ser criada por resfriamento, evaporação, adição de anti-solvente ou por precipitação (Martínez et al., 2008). Como as curvas obtidas foram achatadas e/ou íngreme (Figura 1) pode-se usar cristalização por anti-solvente, ou precipitação física, facilitando-se a formação de cristais de tamanho médio, podendo ocorrer nucleação primária ou secundária (Martínez, 2005).

As curvas de solubilidade do xilitol em água e água:etanol (1:1) podem ser aproximadas por polinômios de segundo grau e a curva de solubilidade do xilitol em água:isopropanol (1:1) por uma função exponencial, sendo essa funções apresentadas na Tabela 1.

Tabela 1: Ajuste polinomial e coeficiente de correlação das curvas de solubilidade em diferentes solventes.

\begin{tabular}{ccc}
\hline Solução & Função & $\begin{array}{c}\text { Coeficiente de } \\
\text { regressão }\end{array}$ \\
\hline Água & $X^{\text {Sat }}=-0,0082 T^{2}-0,0764 T+58,201$ & $R^{2}=0,9998$ \\
Água/Etanol (1:1) & $X^{\text {Sat }}=-0,007 T^{2}+1,7904 T-13,149$ & $R^{2}=0,9793$ \\
Água/Isopropanol (1:1) & $X^{\text {Sat }}=4,1389 e^{0,052 T}$ & $R^{2}=0,9433$ \\
\hline
\end{tabular}


O conhecimento da temperatura de saturação referente a uma determinada fração mássica é importante para determinar em que temperatura se iniciará a zona metaestável, para "semeação" do meio com cristais de xilitol (Wei et al., 2010), sendo essas curvas utilizadas no processo de cristalização.

\subsection{Produção do Xilitol}

A concentração de xilitol obtida no bioprocesso utilizando a levedura $K$. marxianus CCA510 no meio semissintético foi aproximadamente $13,90 \mathrm{~g} / \mathrm{L}$. A levedura consumiu praticamente toda a xilose presente no meio, permanecendo $1 \mathrm{~g} / \mathrm{L}$ após $72 \mathrm{~h}$ de cultivo, e nas condições avaliadas não detectou-se a produção de etanol.

\subsection{Tratamento do meio cultivado}

Para remoção de impurezas presente no meio cultivado e para remoção de pigmentos, foi empregado um processo de adsorção em leito fixo. $\mathrm{O}$ meio passou primeiramente por uma coluna contendo a resina Dowex monosphere 99Ca (Sigma-Aldrich), em seguida por uma resina Diaion UBK555 (Sigma-Aldrich). A Tabela 2 mostra a concentração dos componentes presentes no meio e o $\mathrm{pH}$ após cada processo de tratamento.

Tabela 2: Caracterização e pH do meio cultivado livre de células após o processo de tratamento com resinas de troca iônicas.

\begin{tabular}{ccccc}
\hline Componente & Meio Cultivado & $\begin{array}{c}\text { Após a resina } \\
\text { Dowex 99Ca }\end{array}$ & $\begin{array}{c}\text { Após a resina } \\
\text { Diaion UBK 555 }\end{array}$ & $\begin{array}{c}\text { Retenção total } \\
\text { nas colunas }(\%)\end{array}$ \\
\hline Xilitol $(\mathrm{g} / \mathrm{L})$ & 13,90 & 4,73 & 3,25 & 80,05 \\
Xilose $(\mathrm{g} / \mathrm{L})$ & 1,00 & 0,00 & 0,00 & 100 \\
Ácido acético $(\mathrm{g} / \mathrm{L})$ & 2,69 & 1,47 & 0,55 & 82,37 \\
pH & 5,13 & 5,13 & 4,98 & - \\
\hline
\end{tabular}

Com o processo de adsorção, o meio cultivado foi clarificado, devido substâncias orgânicas ficarem retidas nas colunas (Wei et al., 2010; Seppälä et al., 2010), e observou uma retenção de $100 \%$ de xilose na primeira resina utilizada. Porém, houve uma baixa seletividade, pois o processo também removeu $80 \%$ do xilitol produzido.

Para que se atingisse uma concentração de xilitol favorável ao processo de cristalização, o meio bioprocessado foi concentrado por evaporação até um volume final de $20 \mathrm{~mL}$ a $65{ }^{\circ} \mathrm{C}$, correspondendo a uma concentração de 36 vezes. O meio resultante deste processo teve a concentração de 100,80 g/L de xilitol e não observou-se a presença de ácido acético.

\subsection{Cristalização e Análises dos Cristais de Xilitol}

Ao término de 24 horas de cristalização, foi detectada a formação de uma fase heterogênea com um precipitado coloidal. As fases foram separadas e submetidas à análise. A Tabela 3 apresenta a massa de xilitol nas duas fases obtidas no processo de cristalização, assim como os rendimentos de cristalização. 
Tabela 3: Distribuição mássica do xilitol entre as fases nos processos de cristalização.

\begin{tabular}{cccccc}
\hline \multirow{2}{*}{ Xilitol } & $\begin{array}{c}\text { Álcool } \\
\text { utilizado na } \\
\text { cristalização }\end{array}$ & $\begin{array}{c}\text { Temperatura de } \\
\text { adição dos } \\
\text { cristais }\left({ }^{\circ} \mathrm{C}\right)^{\mathrm{i}}\end{array}$ & $\begin{array}{c}\text { Massa de } \\
\text { Xilitol } \\
\text { Cristalizada }(\mathrm{g})\end{array}$ & $\begin{array}{c}\text { Massa de xilitol } \\
\text { permanecendo no } \\
\text { meio }(\mathrm{g})\end{array}$ & $\begin{array}{c}\text { Rendimento } \\
\text { cristalização } \\
(\%)\end{array}$ \\
\hline Xilitol & Etanol & 35 & 0,5292 & 0,4788 & 52,5 \\
Produzido & Isopropanol & 43 & 0,5574 & 0,4506 & 55,3 \\
\hline Xilitol & Etanol & 35 & 4,26 & 3,74 & 53,3 \\
(Sigma) & Isopropanol & 43 & 4,50 & 3,50 & 56,4 \\
\hline
\end{tabular}

i) Temperaturas determinadas utilizando as curvas de solubilidades apresentadas na Tabela 1.

Os dois anti-solventes apresentaram rendimentos de cristalização bem próximos, obtendose o maior rendimento (55\%) utilizando o álcool isopropanol como anti-solvente. O processo de cristalização utilizando o xilitol PA apresentou o mesmo perfil, indicando que o processo de cristalização pode ser empregado para o meio bioprocessado e tratado. No entanto, os cristais obtidos no processo com xilitol PA foram maiores comparados com os cristais do xilitol produzido neste estudo. Resultados semelhantes ou superiores de rendimento de cristalização foram obtidos por Canilha et al. (2008), Wei et al. (2010) e Misra et al. (2011). No entanto, esse autores utilizaram soluções supersaturado $(750 \mathrm{~g} / \mathrm{L})$ e esse fator aumenta o processo de nucleação (Misra et al., 2011).

Os cristais obtidos nos dois sistemas de cristalização apresentaram pureza de $94 \%$. Resultados semelhantes foram obtidos por Wei et al. (2010), que estudaram a cristalização de xilitol obtivo por bioprocesso usando resíduos hemicelulósicos e alcançaram, a partir de uma solução concentrada com $750 \mathrm{~g} / \mathrm{L}$ de xilitol, um caldo com xilitol de $95 \%$ de pureza e 60,2\% de rendimento de cristalização de xilitol.

As Figuras 2 e 3 apresentam as formas assumidas pelos cristais de xilitol obtidos no bioprocesso após o processo de cristalização utilizando o etanol e isopropanol como antisolventes, respectivamente. Observando-se os cristais apresentados nas Figuras 2 e 3, verifica-se que ocorreu prevalência de partículas pequenas. Nota-se o aparecimento de vários cristais de tamanho pequenos, ao invés de cristais maiores, assim a cristalização ocorreu com velocidade de resfriamento muito rápida, interferindo no desenvolvimento dos núcleos já existentes, que caracteriza a formação de núcleos secundários. $\mathrm{O}$ crescimento de cristais, que está associado à nucleação, é uma etapa complexa (Mullin, 2001).

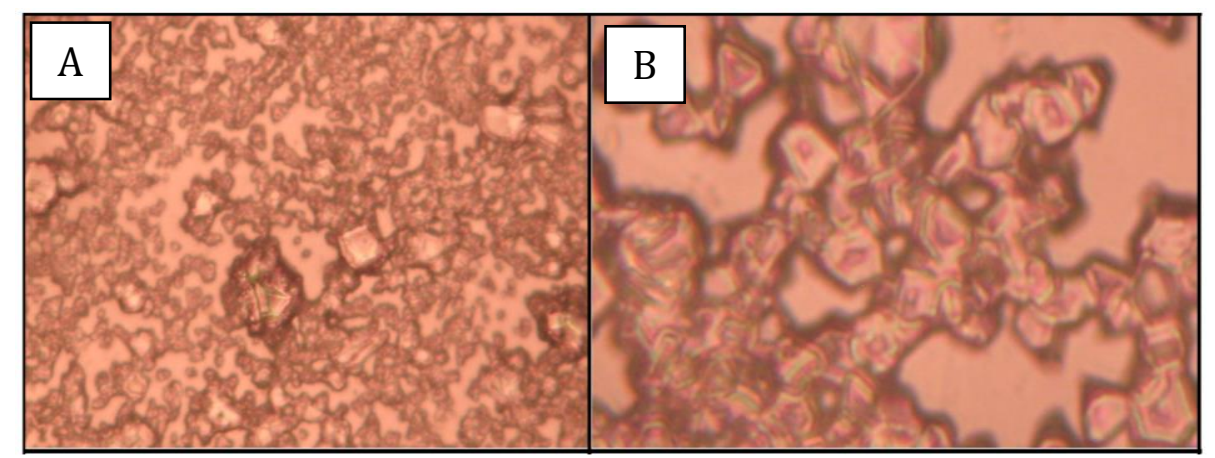

Figura 2: Microscopia dos cristais de xilitol obtido no processo de cristalização utilizando etanol como anti-solvente, ampliada 80x (A) e 440x (B). 


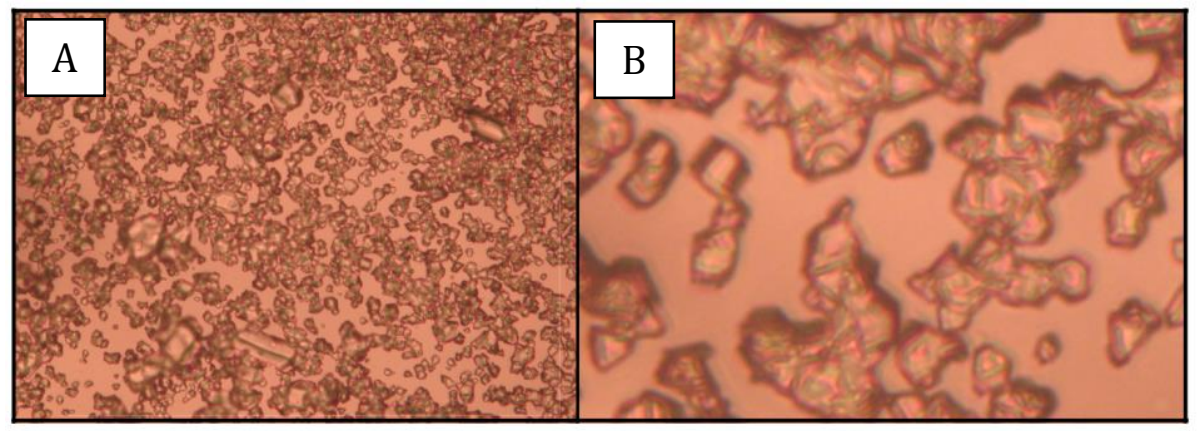

Figura 3: Microscopia dos cristais de xilitol obtido no processo de cristalização utilizando isopropanol como anti-solvente, ampliada 80x (A) e 440x (B).

O sistema de cristalização com o álcool isopropílico apresentou a formação de cristais com tamanho ainda menor do que o sistema de cristalização com o etanol conforme Figuras 2 e 3. Em ambos os casos os cristais produzidos foram bem menores do que o cristal de xilitol comercial (Sigma). Hao et al. (2006) determinaram a solubilidade de xilitol no sistema águametanol, concluindo que a solubilidade, a velocidade de nucleação e da taxa de crescimento de cristais de xilitol diminuíram com o aumento do teor de metanol e que a taxa de resfriamento é um dos fatores que influência na formação dos cristais.

\section{CONCLUSÕES}

No processo de tratamento do meio cultivado, observou-se que as resinas de troca iônica empregadas, apesar de eficientes na clarificação do meio, ocasionaram grande perda de xilitol, e por isso, é necessário avaliar outras resinas. No processo de cristalização, etanol e isopropanol apresentaram a mesma eficiência como anti-solventes, obtendo rendimentos de cristalização $55 \%$, com cristais apresentando uma pureza de $94 \%$.

\section{REFERÊNCIAS}

AHMAD, I.; SHIM, W.Y.; KIM, J.-H. Enhancement of xylitol production in glycerol kinase disrupted Candida tropicalis by co-expression of three genes involved in glycerol metabolic pathway. Bioproc. Biosyst. Eng., v. 36, p. 1279-84, 2013.

ALBUQUERQUE, T. L. Produção biotecnológica de xilitol a partir de hidrolisado de bagaço de caju. 2014. 148f. Dissertação (Mestrado em Engenharia Química, Departamento de Engenharia Química), Universidade Federal do Ceará, Fortaleza, 2014.

BELTER, P.; CUSSLER, E.L.; HU, W. Bioseparation: Dowstream Processing for Biotechnology, John Wiley \&Sons, 1988. 368p.

BURNEO, M. C. G. Produção de xilitol a partir de leveduras isoladas de madeira em decomposição do arquipélago das Ilhas Galápagos (Equador). 2013. 124f. Dissertação (Mestrado em Microbiologia) - Instituto de Ciências Biológicas. Universidade Federal de Minas Gerais, Belo Horizonte, 2013.

CANIlHA, L.; CARVAlho, W.; GIUliETTI, M., FELIPE, M.G.A, ALMEIDA E 
SILVA J.B. Clarification of a wheat straw-derived medium with ion exchange resins for xylitol crystallization. J. Chem. Technol. Biotechnol. v. 83, p. 715-721, 2008.

DERENZO, S. Estudo da cristalização de ácido adípico em batelada, por resfriamento e em escala de laboratório. Dissertação de Mestrado. 162f. EPUSP, São Paulo, 1994.

HAO, H.; HOU, B.; WANG, J-K.; LIN, G. Effect of solvent on crystallization behavior of xylitol. J. Crystal Growth, v. 290, p. 192-196, 2006.

MARTÍNEZ, E. A. Estudo do processo de cristalização de xilitol obtido por via fermentativa. 2005. 175f. Tese (doutorado) - Faculdade de Engenharia Química de Lorena, Lorena, 2005.

MARTÍNEZ, E. A.; GIULIETTI, M.; SILVA, J. B. A.; DERENZO, S. Kinetics of the xylitol crystallization in hydro-alcoholic solution. Chem Eng Proc., 47, 2157-2162, 2008.

MARTÍNEZ, E. A.; GIULIETTI, M.; SILVA, J. B. A.; DERENZO, S.; FELIPE, M. G. A. Batch cooling crystallization of xylitol produced by biotechnological route. $J$ Chem. Technol. Biot., v. 84, 376-381. 2009.

MISRA, S.; GUPTA, P.; RAGHUWANSHI, S.; DUTT, K.; SAXENA, R. K. Comparative study on different strategies involved for xylitol purification from culture media fermented by Candida tropicalis. Sep. Purif. Technol., v. 78, n. 3, 266-273, 2011.

MULLIN, J.W.: Crystallization, quarta edição, London, England, Butterworth Heinemann, 2001.

SARROUH, B. F. Estudo da produção biotecnológica de xilitol em reator de leito fluidizado utilizando bagaço de cana-de-açúcar e células imobilizadas: avaliação de parâmetros operacionais e viabilidade econômica. 2009. 185f. Tese (Doutorado em Biotecnologia Industrial) - Escola de Engenharia de Lorena da Universidade de São Paulo, Lorena, 2009.

SEPPÄLÄ, A.; MERILÄINEN, A.; WIKSTRÖM, L.; PAURANEN, P. The effect of additives on the speed of the crystallization front of xylitol with various degrees of supercooling. Experimental Thermal and Fluid Science, v. 34, 523-527, 2010.

SOLEIMANI, M.; TABIL, L. Evaluation of biocomposite-based supports for immobilizedcell xylitol production compared with a free-cell system. Biochem. Eng. J., v. 82, 166-173, 2014.

WEI, J.; YUAN, Q.; WANG, T.; WANG, L. Purification and crystallization of xylitol from fermentation broth of corncob hydrolysates. Frontiers Chem. Eng. China, v. 4, n. 1, 57-64, 2010 . 